\title{
Review: Link between intestinal immunity and practical approaches to swine nutrition
}

\author{
B. Humphrey ${ }^{1 \dagger} \oplus$, J. Zhao ${ }^{2}$ and R. Faris ${ }^{2}$ \\ ${ }^{1}$ Provimi, 10 Nutrition Way, Brookville, OH 45309, USA; ${ }^{2}$ Cargill Animal Nutrition, 10383 165th Ave NW, Elk River, MN 55330, USA
}

(Received 15 March 2019; Accepted 19 July 2019; First published online 2 September 2019)

\begin{abstract}
Gaining a deeper understanding into the underlying mechanisms associated with intestinal function and immunity during the weaning transition is critical to help shed new light into applied nutrition approaches to improve piglet performance and health during this critical life-stage transition. The transient anorexia triggered at weaning leads to compromised intestinal barrier function and a localized inflammatory response. Considering barrier function, specific nutrient fractions appear to have a significant impact on the development and function of the immune and microbial systems around weaning. Understanding the specific impact of nutrients in the small intestine and hindgut is important for helping to bring more focus and consistency to nutritional approaches to support health and immunity during the weaning transition period. The challenge continues to be how to translate these modes of action into practical and scalable approaches for swine nutrition. We will focus specifically on practical nutritional approaches to influence intestinal immunity through lipid, protein and antioxidant nutrition.
\end{abstract}

Keywords: swine, nutrition, immunity, intestine, weaning

\section{Implications}

The ability to translate the physiological, immunological and digestive changes at weaning into practical nutritional approaches for commercial swine production remains an exciting challenge for our industry. As we continue to gain more insight into the underlying modes of action, a more targeted nutrient approach can be applied to help achieve more targeted and consistent outcomes for the weaned pig.

\section{Introduction}

Gaining a deeper understanding into the underlying mechanisms associated with intestinal function and immunity during the weaning transition is critical to help shed new light into practical nutrition approaches to improve piglet performance and health during this critical life-stage transition. Nutrition approaches have focused on achieving heavier weaning weights by providing highly digestible diets to encourage better DM intake as the young pig transitions from milk to solid feed. The benefits of achieving success in the weaning transition are significant. Tokach et al. (1992) demonstrated that heavier weaning weights and higher gain

\footnotetext{
${ }^{\dagger}$ E-mail: bhumphrey@provimi-na.com
}

during the first-week post-weaning can result in a 10-lb BW advantage at day 56 of age and up to a 10-day marketing advantage. Main et al. (2004) demonstrated that the potential improvement of increasing weaning age by 1 day between day 12 and 21 would result in an extra $0.93 \mathrm{~kg}$ of day $42 \mathrm{BW}$ and up to $0.47 \%$ reduction in wean-to-finish mortality. The economic implications of these aforementioned benefits, among others, are significant to swine producers and will continue to drive urgency to find new ways to achieve these advantages in commercial settings. Intestinal function and immunity during the weaning transition are critical leverage points for the ability of nutrition to influence immediate and longer term performance and health impacts (Jacobi and Odle, 2012). Consequently, there is a need to gain better insights to underlying physiological, nutritional and immunological adaptations occurring in the intestine at weaning to allow for practical application of nutritional approaches to support immunity and intestinal function.

\section{Intestinal immunity during the weaning transition}

The development of the gastrointestinal system occurs over several months after birth. Development of digestive functions coincides with the development of the adaptive and innate immune systems. From an immune development 
standpoint, the innate immune system defenses responsible for barrier function are more mature relative to the adaptive immune system given the time needed for lymphocytes to populate secondary lymphoid tissues, mature and expand in order to function properly (Bailey et al., 2005). Piglets are limited in their ability to mount $T$ and $B$ cell responses during the first few weeks of life, and this contributes to an overall immuno-compromised condition (Lalles et al., 2007). Consequently, the young pig is more reliant on innate immunity given the maturity of this system through the first few weeks of life. The intestinal immune system provides protection along the intestinal tract and balances the host response to microflora dynamics and intestinal pathogens. The structure and function relationships of barrier function and intestinal immunity development have been reviewed in detail (Everaert et al., 2017; Moeser et al., 2017). The type and rate of microbial colonization in the intestine and the host's response to their presence play an important role in the establishment of intestinal immunity.

The intestinal microbiome ecosystem is extremely complex and is tightly linked to performance and health. The changes in the microbiome ecosystem in pigs are more variable and less stable relative to adults, which can pose a health and performance risk that may have long-term impacts (Merrifield et al., 2016). The changes in microflora at weaning are broadly characterized by a reduction in obligate anaerobic bacteria (e.g., Clostridia and Bateroidia) and an increase in facultative anaerobic bacteria (e.g., Enterobacteriaceae) which translate to decreased microbial diversity and a pro-inflammatory state within the intestine (Gresse et al., 2017). While certainly not mutually exclusive, changes in the microbiome ecosystem are also influenced by nutritional and husbandry approaches. For example, piglets raised on milk replacers have significantly different microbial ecosystems and functional intestinal immune responses relative to piglets raised with the sow (Lewis et al., 2012). Additionally, while sow genetics can also influence the development of the piglet microbial ecosystem, the age of weaning and time of exposure to solid feed have a greater impact (Bian et al., 2016). Taken together, the microbiome ecosystem is dynamic and we are continuing to learn how this can be impacted by nutrition, genetics and husbandry. This continues to be an area where a deeper understanding of how microbial phenotypes translate to practical outcomes in commercial settings is still needed. While there is progress to begin to understand some relationships, such as linking porcine gut microbial ecosystems and performance (RamayoCaldas et al., 2016), there will be a need to understand how specific nutrients and additives, such as prebiotics, probiotics, phytogenics, enzymes, among others, can more consistently make the desired impacts through microbiome mode of action. While not intended to go into full detail in this review, the impact of specific additives on intestinal immunity and microbiome ecosystems has been discussed (Lalles et al., 2007; Jacobi and Odle, 2012; Gresse et al., 2017). Additionally, as the identification of preferred microbiome modifiers is better understood and appreciated we can begin to assess different nutritional approaches to help improve transition from sow milk to dry feed, better productivity and health.

\section{Nutritional implications of an intestinal immune response}

Pastorelli et al. (2012) completed a meta-analysis to understand the impact of an immune response on feed intake and growth responses. The meta-analysis classified the performance response to immunity according to digestive bacterial infections, poor housing conditions, lipopolysaccharide challenge, mycotoxicosis, parasitic infections or respiratory disease. Digestive bacterial infections had the greatest negative impact on growth response relative to the other categories evaluated. The change in gain was partly explained by a reduction in intake; however, the majority was attributed to reduced feed efficiency and changing maintenance requirements and digestive function during the immune challenge. Intestinal immune responses result in a significant nutritional cost to the animal as shown through reductions in intake and the efficiency in which these nutrients are used for growth. While not a pathogenic challenge, the combination of changes in intestinal architecture, microbial profiles and immune-competence of the pig at weaning initiates an intestinal immune response that can contribute to limitations in performance and health during the weaning transition (Lalles et al., 2007). As we continue to understand the morphological, physiological, endocrine and immune mechanisms that contribute to these phenotypes, we can improve our ability to target nutrition to help mitigate these limitations encountered at weaning. Intestinal barrier function continues to be the focal point for nutritional support given the morphological and endocrine changes that occur during the weaning transition period.

Intestinal barrier function plays a critical role in the overall health and performance of weaned pigs (Moeser et al., 2017). The combined social, microbial and environmental stressors at weaning lead to targeted impacts on barrier function due to reductions in intake. Reductions in intake at weaning are significant and in some instances it may take 10 to 14 days to reach pre-weaning intake levels (Le Dividich and Seve, 2000). The reduction in energy intake at weaning reduces villous height and increase crypt depth, which collectively contributes to reduced enzymatic capacity and absorptive surface area (Le Dividich and Seve, 2000). By providing more energy intake at weaning through supplementation of milk, reductions in villous height can be mitigated, suggesting that feed intake and beneficial intestinal histology have a positive association (Le Dividich and Seve, 2000). Changes in morphology of the intestine occur relatively quickly compared to intestinal barrier function, and this may explain why histological measurements do not always associate well with performance metrics. As noted by Moeser et al. (2017) and mentioned earlier with the meta-analysis of digestive bacterial infections, a 
breakdown in intestinal barrier function can have deleterious consequences on health and animal performance.

The weaning process results in increased intestinal permeability due to decreased tight junctions (Moeser et al., 2007). This leads to transmigration of luminal antigens, toxins, viruses or even bacteria into the lamina propria. These pathogen-associated molecular patterns are well recognized by cells of the immune system that reside within the lamina propria, and their activation results in the release of pro-inflammatory mediators and signaling molecules to help neutralize and recruit other immune cells into the region (Lalles et al., 2007). This results in an inflammatory response, which can further contribute to reductions in intake and reduced growth. The inflammatory response results in a catabolic response and, depending on the severity and time frame of the inflammatory response, a shift in the flux of nutrients away from growth and more toward immune support (Klasing and Johnstone, 1991). Skeletal muscle catabolism helps to support amino acid substrate for the synthesis of acute phase proteins that are an important component of the innate immune response to infection. While the reductions in animal performance during an immune response are largely associated with decreased feed intake, the increase nutrient need for the immune system may help explain why digestive bacterial infections resulted in the greatest reduction in growth relative to other immune challenges (Pastorelli et al., 2012). Consequently, nutritional approaches to support barrier function and/or modified inflammatory response can help mitigate the negative impacts of weaning on health and performance.

Weaning stress also results in activation of the hypothalamicpituitary adrenal axis, resulting in elevated serum corticotrophinreleasing factor (CRF) for days post-weaning (Moeser et al., 2007). In the intestine, CRF plays a major role in stress-related intestinal disturbances and does so by binding to CRF receptors on epithelial, neuron and immune cells, most notably mast cells (Bonaz and Bernstein, 2013). Mast cell degranulation within the intestine results in inflammatory mediators to help destroy pathogens and signaling molecules, such as chemokines, and other regulators such as interleukins that will help to coordinate the local immune response. In fact, administration of the mast cell stabilizer cromolyn prevented weaning-induced increases in intestinal permeability (Moeser et al., 2007) and increased postweaning growth to 36 days of age by $20 \%$ (Mereu et al., 2015). These data show the importance of the mast cell in the weaninginduced changes in intestinal barrier function and the potential that can exist when steps in these processes can be ameliorated. Taken together, while there is certainly more knowledge needed in these areas, practical nutrition approaches focused on supporting barrier function have the potential to help mitigate local and systemic inflammatory responses.

\section{Nutritional approaches to support barrier function}

As mentioned previously, the transient anorexia triggered at weaning leads to compromised intestinal barrier function and a localized inflammatory response. The absence of food in the intestine leading to these changes in intestinal function and immunity points to the importance of feed intake at weaning. In neonatal pigs, data suggest that a minimum of $40 \%$ of total nutrient intake is required to maintain intestinal mucosa and $60 \%$ of total nutrient intake is required for increased intestinal growth and proliferation (Burrin et al., 2000). Simple measures to help promote intake are a very practical step to help ameliorate these inflammatory responses and improve barrier function (Pluske et al., 1997; McCracken et al., 1999). As reviewed by Anastasilakis et al. (2013), the absence of nutrition stimulus from food in the gastrointestinal tract (GIT) results in changes in intestinal immunity. This includes a reduction in intestinal immune cells and imbalances in cytokines, chemokines and other immune markers. Of note, these changes include IL-4, IL-10 and IFN $\gamma$ leading to a more prominent T-helper 1 phenotype. A subsequent effect of this shift in immune profile is decreased IgA content in intestinal and mucosal tissues. Changes in intestinal microflora also occur as a consequence of no enteral intake. Compared to enteral nutrition, parenteral nutrition changes the intestinal microflora to predominately gram-negative Proteobacteria (Demehri et al., 2013), a phylum containing a number of pathogens including Escherichia and Salmonella. This shift to Proteobacteria is linked to increased toll-like receptor (TLR) signaling in the intestine, which can further increase inflammation and decrease intestinal barrier function. Blocking of TLR signaling through the use of MyD88 knockout mice inhibits the increase in TNF $\alpha$ and IFN $\gamma$ and mitigates the effect from the shift to Proteobacteria (Demehri et al., 2013). Consequently, promoting early intake and weaning and more consistent meal patterns at weaning are important to ensure the presence of feed in the intestine to further mitigate inflammation.

A central focus of nutrition for early intake at weaning continues to be on nutrient use and availability given the importance of animal performance, health and sustainability. Considering barrier function and intestinal immunity, however, what is undigested and how that is influencing microbial changes within the intestine may be just as important. This continues to be an area of opportunity as we understand the impacts of nutrient release into the distal small intestine and hindgut and how these can influence immunity, health and feeding patterns during the weaning transition period. The opportunity continues to be how to translate these into practical approaches for swine nutrition and building health outcome-based diets, such as improved livability and reduced medication need, for pigs during the weaning transition. We will focus specifically on practical nutritional approaches to influence intestinal immunity through lipid, protein and antioxidant nutrition. The impact of carbohydrates on intestinal health has been reviewed (Bach Knudsen et al., 2012), and the specific impact of insoluble and soluble fiber fractions on barrier functions continues to be evaluated (Wu et al., 2018). From a practical nutrition standpoint, we will assess different approaches to promote 
intestinal barrier function through delivery of lipids as signaling molecules, control of undigested protein and ability to support antioxidant defense during the weaning transition period.

\section{Lipid nutrition}

Fat is the primary energy source during the first few weeks of life. Weaning reduces fat calorie intake given the shift to carbohydrate-based ingredients. The impact of fat calorie intake on satiation and meal patterns in piglets is not well investigated. Lipid research for weaned pigs has mainly focused on comparing different types of fats and oils on performance, digestibility and other traits (Cera et al., 1988; Kim et al., 2013), n-3 fatty acids for their antiinflammatory potential (Carroll et al., 2003) or medium chain fatty acids or triglycerides as readily available source of energy with antimicrobial potential (Hanczakowska, 2017). Less focus has been given to the effect of lipids on gastrointestinal transit and peptide secretion and effect on intake and intestinal health. Several studies investigated the effect of lipid infusion in the pig gastrointestinal tract and its effect on intake and Cholecytokinin (CCK). Gregory and Rayner (1987) investigated the effects of stomach, duodenum, jejunum or ileum infusion of intralipid or digestion products on feed intake. When intralipid was infused in the duodenum, it dose dependently decreased feed intake to a greater extent than the energy infused. Infusion of monoglyceride, oleic acid and glycerol also had effects on intake when infused in the duodenum or ileum, but only infusion of monoglyceride decreased the overall energy intake. Further experiments determined that CCK mediated the effects on intake when intralipid or monoglyceride was infused in the duodenum (Gregory et al., 1989b) and that duodenal infusion of intralipid and glucose inhibits gastric emptying (Gregory et al., 1989a). Collectively, the data indicate that lipids influence gastric emptying and that the intestinal location of lipid digestion products can also influence intake and gastrointestinal peptide secretion.

More recent efforts in humans have investigated the effect of lipid gastric emulsion stability and droplet size on gastric emptying, gastrointestinal peptides and satiety. Foltz et al. (2009) investigated the effect of feeding a meal that was mixed with fat (i.e., emulsion) or non-mixed (i.e., layered) on intake responses. The layered fat delayed lipid absorption, increased post-prandial CCK and increased satiety. Differences between acid stable and acid unstable emulsions have also been investigated (Marciani et al., 2007 and 2009). Acid unstable emulsions quickly separate in the stomach leading to fat layering and decreased gastric emptying, increased post-prandial CCK and an increased feeling of satiety. These results are in contrast to the results of Foltz et al., 2009. Differences may be attributed to how the meal was delivered and the quantity of fat delivered in the two experiments. Decreasing fat droplet size has similar effects as acid stability on gastric emptying; however, it further increases satiety or decreases subsequent meal size (Hussein et al.,
2015). Further investigations into the effect of differences in gastric emptying on gastrointestinal peptides determined that increased gastric emptying from acid-unstable emulsions increases CCK and Glucagon-like peptide-1 (GLP-1) while lowering total post-prandial CCK. In contrast, increasing droplet size decreased the initial peak in CCK (Steingoetter et al., 2017). Additionally, acid unstable emulsions and increased droplet size decreased total peptide YY, another peptide involved in regulation of intake.

Glucagon-like peptide-1 along with Glucagon-like peptide 2 (GLP-2) is produced by enteroendocrine cells in the intestine and is synthesized from the proglucagon gene and is involved in stimulating intestinal growth, stimulation of digestive activity and nutrient absorption, improvement in intestinal barrier function, regulation of feed intake, improvements in insulin sensitivity and increasing $\beta$-cell proliferation (Brubaker and Anini, 2003; Burrin et al., 2003). Fat is a strong stimulator of GLP-1 secretion (Knapper et al., 1995) by products of lipid digestion including fatty acids (Voortman et al., 2012) and monoglycerides (Hansen et al., 2011). While prior data have focused on the effect of lipids regulating intake, in cases of low feed intake, such as weaned pigs, the effect of lipids on gastrointestinal peptide secretion and the impact on intestinal barrier function and potential for immune modulation may be as relevant as the impact on intake. Treatment with GLP-2 decreases serum amyloid $A$ and haptoglobin concentrations in the blood providing evidence that it can reduce inflammation in conditions of low intake (Kvidera et al., 2017). Like GLP-2, there is also evidence of anti-inflammatory effects of GLP-1 for a number of chronic conditions in humans (Lee and Jun, 2016). Translational data in pigs are currently limited; however, these provide new perspectives into lipid nutrition for young pigs to help support intestinal barrier function and immunity through improved feed intake.

\section{Undigested protein}

Undigested protein can be defined as the amount of $\mathrm{N}$ that enters into the hindgut for use in bacterial fermentation. This $\mathrm{N}$ source is in excess of what the pig is able to digest and absorb, either due to poor protein quality and/or limited $V_{\max }$ of digestive enzymes and nutrient transporters in the intestine. The excess $\mathrm{N}$ available for fermentation in the distal ileum and colon has a negative impact on barrier function and immunity and is a major contributor to post-weaning diarrhea at weaning (Kim et al., 2012). Increasing total $\mathrm{N}$ flow into the terminal ileum results in increased $\mathrm{NH}_{3}-\mathrm{N}$ content in the ileum and hindgut as well as increased $\mathrm{pH}$ (Heo et al., 2010a and 2010b). Increased $N$ availability results in a shift in microbial populations toward more proteolytic populations and increased production of fermentation byproducts, such as branched chain fatty acids, ammonia, amines, volatile phenols and indols (Williams et al., 2001). Microbial populations shift from Lactobaccilus spp and butyrate producing saccharolytic microbes to more proteolytic spp such as Clostridium (Opapeju et al., 2009; Gresse et al., 2017). 
Products of protein fermentation have toxic and pro-inflammatory impacts on the intestinal epithelium. Consequently, undigested protein is a substrate that leads to more toxic compounds in the intestinal lumen during a time when the intestinal tract is immature and more susceptible to the negative impacts of these compounds. To further compound the issue, pathogen challenges, which are common in commercial settings, further exacerbate these responses. A pathogen challenge, such as enterotoxigenic Escherichia coli, increased total $\mathrm{N}$ flow to the distal ileum, further increases $\mathrm{pH}$ and has more of an impact on intestinal structure, such as villous height and associated impacts on nutrient digestion and absorption potential (Heo et al. 2009 and 2010b; Pluske et al., 1997). In addition, toxins produced by pathogens such as $E$. coli can also disrupt water regulation and absorption and reabsorption of minerals, which contributes to postweaning diarrhea (Sun and Kim, 2017). These impacts, both undigested protein and pathogen infection, lead to an inflammatory response in the intestine that can have systemic implications and may further reduce performance (Pieper et al., 2012). There is an opportunity, therefore, to apply nutritional approaches to mitigate the negative impacts of undigested protein on intestinal barrier function in order to support a smooth transition through weaning.

Reductions in dietary CP levels in post-weaning diets have long been advised (Nyachoti et al., 2006; Kim et al., 2012). Interestingly, while digestive enzymes of the weaned pig are compromised at weaning, the apparent ileal digestibility is not impacted by CP level or during an $E$. coli infection, suggesting that $V_{\max }$ for these digestive processes has been achieved at even $20 \%$ to $40 \%$ reduction in dietary CP levels. Total $\mathrm{N}$ flow to the terminal ileum in either low- or high-CP diets did differ, however, which was mainly due to similar intake across these diet types (Heo et al. 2009 and 2010b). These data suggest that high-CP diets, such as in excess of $23 \% \mathrm{CP}$ as fed in these studies, under health and low disease pressure may not be detrimental; however, from a commercial perspective where disease challenge is high the ability to reduce total $\mathrm{N}$ flow through lower CP diets can help support reduced post-weaning diarrhea (Kim et al., 2012). Lowering the dietary protein content with balanced AA might reduce the formation of microbial metabolites and consequently reduce the incidence of diarrhea. Htoo et al. (2007) showed no effect on piglet performance and fecal score, ileal ammonia and ileal amine content when the protein level of the diets was lowered from $24 \%$ to $20 \%$ with the same levels of standardized ileal digestibility (SID) AA and energy.

While there continues to be a need to gain better insight into amino acid availability and nutrition across life stages and feedstuffs, more focused attention on amino acid needs for the intestine are somewhat limited. The long-standing practical nutrition principle is to utilize more expensive specialty proteins in piglet feeds and to maintain the proper amino acid ratios (Goodband et al., 2014). There continues to be interest in evaluating amino acid ratios for optimal intestinal barrier function at weaning. Specific attention has been focused on tryptophan given the further metabolism of this amino acid to functional metabolites that can have immuneregulatory properties, such as regulation of $\mathrm{T}$ cell function and response. Results with tryptophan are somewhat unclear, as Le Floc'h et al. (2009) reported lower plasma Trp levels suggesting that Trp availability was limited for growth and other metabolic functions. Capozzalo et al. (2012) discovered that Trp:Lys ratios in excess of growth resulted in improved feed efficiency in either the presence or absence of an enterotoxigenic $E$. coli infection; however, there was no immune challenge by Trp:Lys interaction. Optimal Thr:Lys ratios have also been evaluated in piglets given the importance of this essential amino acid for intestinal use and barrier function (Stoll et al., 1998). Wang et al. (2010) demonstrated that true ileal digestibility Thr levels of $0.37 \%$ or $1.11 \%$ were least effective for villous architecture and concentrations of ileal acidomucins and duodenal sulfomucins. The optimal ratio of Thr:Lys for supporting intestinal barrier function was concluded to be $0.89 \%$ for weaning piglets, which was above the optimum level of $0.74 \%$ for growth performance (Wang et al., 2010). Dispensable amino acids also play an important role in intermediary nitrogen metabolism through production of conditionally essential amino acids (e.g., arginine), energy metabolism intermediates (e.g., tricarboxylic acid (TCA) cycle intermediates) and also purines and pyrimidines. Collectively, these positively impact energy status in the intestine, which can lead to enhanced integrity and function (Burrin and Stoll, 2009). Amino acids that seem to be of particular importance are glutamine, glutamate, proline, aspartate, ornithine and citrulline, which is due in large part to their connectivity via inter-organ amino acid metabolism. Of particular importance is the ability of these amino acids to positively regulate AMP-activated protein kinase (AMPK), which is the master regulator of cellular energy status. Glutamate, glutamine and aspartate are primary energy substrates for the intestine and have been shown to modify AMP:ATP ratios in the intestine, which can influence AMPK signaling pathways. The ability to fully leverage these insights is somewhat limited today, mainly due to commercial limitations associated with synthetic amino acid price and sourcing along with analytical limitations of the availability of these amino acids in different raw materials. Most ideal protein studies have assessed the appropriate ratio to lysine to other indispensable amino acids, but have not determined the optimal ratio of indispensable to dispensable amino acids. The ratio of indispensable to dispensable amino acids at a given dietary protein level is also important and is determined by the ratio of lysine to protein (Li et al., 2001). Although an average total lysine:CP ratio of $6.8 \%$ is often cited, a higher lysine:CP ratio can be used in the diet because the lysine released during normal muscle protein breakdown is conserved and recycled with greater efficiency than other amino acids (Nemechek et al., 2011). Ratliff cited by Nemechek et al. (2011) suggested that the total Lys:CP ratio should not exceed $7.1 \%$.

Crude protein and amino acid digestibility of raw materials is another important factor contributing to fermentable 
protein and associated challenges to the GIT during the weaning transition. The current approach is to use a similar standardized ileal digestibility value for all life phases. Li et al. (1993) indicated that the ileal amino acid digestibility increases with age and that weaned piglets may have a reduced capacity for $\mathrm{CP}$ and amino acid use. This is also supported by reduced pancreatic and brush border enzymes around the time of weaning. Consequently, the use of SID values for growing pigs may not be adequate for the weaned pig and this may have implications on the amount and variability of undigestible protein. Urbaityte et al. (2009) determined standardized ileal CP and amino acid digestibilities for protein supplements using 15-day-old (BW $4 \pm 0.5 \mathrm{~kg}$ ) ileal cannulated barrows. Compared to tabular grower-finisher values from National Research Council (NRC), SID values for indispensable amino acids were 2 to 19 percentage points lower for extruded soybean meal and 2 to 12 percentage points lower for high-protein soybean meal. These indicate that for some ingredients there is a large difference in SID values, which will result in a larger impact of fermentable protein on intestinal health and immunity. For some high-quality protein ingredients, the ileal digestibilities are relative similar or close to what is recommended in either the NRC or Centraal Veevoeder Bureau (CVB) standards. In this case, the use of high-quality protein as proposed by Goodband et al. (2014) to help mitigate nutritional challenges to the intestine are well supported.

\section{Antioxidant nutrition}

Weaning and weaning-associated intestinal inflammation generate large amount of reactive species. Jejunum reactive oxygen species increase threefold, and thiobarbituric acidreactive substances increase $50 \%$ in 28 -day-old weaned pigs compared with pre-weaning pigs (Wei et al., 2017). Similar results were reported by Zhu et al. (2012) in that the concentrations of nitric oxide and hydrogen peroxide were significantly increased after weaning. Nitric oxide released into the GIT lumen is rapidly transformed into nitrate, which confers a growth advantage for $E$. coli (Gresse et al., 2017). Zeng et al. (2017) remarked that perturbations of the microbiota are commonly observed in diseases involving inflammation in the GIT, with the inflamed microenvironment being particularly conducive to overgrowth of Enterobacteriaceae, which acquire fitness benefits while other families of symbiotic bacteria succumb to environmental changes. A recent study by Wei et al. (2017) reported an increased concentration of reactive oxygen species in the intestine coupled with an expansion of the $E$. coli population 7 days after weaning. Overgrowth of $E$. coli induces further inflammation and activation of the immune system, which in turn generates more free radicals, and undermines its functions (Catoni et al., 2008). Free radical overproduction could overwhelm antioxidant systems and deplete in vivo antioxidants such as vitamin E and enzymes. Kim et al. (2016) reported that plasma vitamin $\mathrm{E}$ was dramatically decreased from around 3.0- to $2.0-\mathrm{mg} / \mathrm{l}$ post-weaning and was further decreased with $E$. coli challenge. Dietary supplementation of $200 \mathrm{IU} / \mathrm{kg}$ of vitamin $E$ is not sufficient to restore plasma vitamin E to pre-weaning status of $3 \mathrm{mg} / \mathrm{l}$.

Another consequence of weaning is dramatic reduction of energy intake. Piglets consume around $1200 \mathrm{~kJ} / \mathrm{BW} 0.75 /$ day from sow milk (Pluske, 1993). A significant percentage of pigs do not eat anything during the first 24- to 72-h postweaning. During this period, body tissue is mobilized to maintain body temperature and essential biological function. Excess tissue mobilization and catabolic status increase free radical production (Bernabucci et al., 2005). This has been described in sows (Zhu et al., 2012), dairy cows (Castillo et al., 2005) and rats with a glucocorticoid-induced catabolic metabolism (Orzechowski et al., 2002). Insufficient energy intake in weaning pigs results in intestine villous atrophy and impaired barrier function. Intestinal inflammation generates great amount of reactive oxygen species (ROS), as indicated previously. It is critical to improve early feed intake to prevent excess body metabolism during the first few days post-weaning, to maintain intestinal integrity and antioxidant balance. Wijtten et al. (2011) reported a linear correlation between villous height and DM intake. Piglets had higher DM intake with longer villous and better barrier function (Pluske et al., 1997).

To protect the cells against potentially damaging effects of radicals, cells possess antioxidant networks that include antioxidant enzymes, such as superoxide dismutase and gluthatione peroxidase, vitamins and non-proteins (coenzyme Q, glutathione). During the weaning period, antioxidant systems are overwhelmed due to intestinal inflammation, pathogenic bacterial overgrowth and catabolic metabolism. Results clearly indicated that intestinal dysfunction after weaning is associated with unbalanced antioxidant systems as indicated by reduced super oxide dismutase (SOD), glutathione peroxidase (GPX) and increased ROS in the intestines and the blood. There is much interest in the use of assessing antioxidant status (Buchet et al., 2017) and compounds (Zhu et al., 2012) to mitigate this problem of antioxidant imbalance. Dietary polyphenols have been shown to play important roles in human health by lowering risks of many chronic diseases including cancer, cardiovascular disease, chronic inflammation and many degenerative diseases (Milner, 1994). Others, such as flavonoids, have direct effects on a variety of immune and inflammatory cell functions (Catoni et al., 2008). Diets supplemented with grape polyphenol extraction at 100 or $150 \mathrm{mg} / \mathrm{kg}$ increased serum IgG, IgM, C4 and IL-2 concentrations (Shi et al., 2003). Combination of vitamin E and C, polyphenols and fermented components effectively improved jejunum and colon antioxidant status (GPX, SOD, total antioxidant (T-AOX)) in weaning pigs (Xu et al., 2014). In addition, intestinal microflora balance is restored with more Lactobacillus and Bifidobacterium and reduced $E$. coli in both the jejunum and the colon compared to weaned controls. Interestingly, a positive correlation of antioxidative status with potential beneficial bacteria and negative correlation 
with E. coliwas reported. These results suggest that intestinal ROS strongly impacts microflora balance which in return impacts intestinal inflammation and antioxidant balance. By scavenging free radicals, or preventing oxidative chain reactions, dietary antioxidant supplements have been shown to improve antioxidant status, immunity as well as restore intestinal microflora balance.

In conclusion, antioxidant balance is a critical aspect of intestinal immunity and microbial balance in weaning pigs. There is a strong relationship between ROS production, microflora population and intestinal immune function. Dietary antioxidants such as vitamins and polyphenols can be used to scavenge ROS and prevent oxidation stress, which consequently improve microflora balance and digestive health.

\section{Conclusions}

Practical nutrition approaches to support intestinal immunity should focus on ways to mitigate the inflammatory response. This requires consideration of not only the nutrient needs for animal performance, but also balancing the nutrient needs impacting the intestinal immune system to ensure resilience and health-based outcomes through the weaning transition. While genetic advancements and husbandry practices will no doubt play a significant role in this advancement, practical nutrition approaches will also be important to ensure that the right intestinal and microbial outcomes are achieved. As discussed in this review, the ability to not only stimulate feed intake post-weaning but rather the intake of specific nutrient fractions that have a more profound impact on achieving health outcomes in weaning is needed. Looking beyond lipids as an energy source and as a regulator of intestinal growth and maturity will cause us to view fat source, quality and application differently. Furthermore, considering what is undigested, in the case of protein, and how this shapes the microbiome and health in the young pig brings a different perspective on how to evaluate protein quality and relative value of protein rich ingredients for young animal feeds. Finally, the weaninginduced increase in reactive oxygen species, as well as the plasma reductions in vitamin $\mathrm{E}$ at weaning, points to an opportunity to consider other sources of antioxidant defense and supplementation. Taken together, there continues to be a need to gain more of an understanding of how to feed the immune system to achieve the desired outcomes in commercial swine production systems. By gaining a deeper insight into the physiological, immunological and digestive changes occurring in the intestine at the time of weaning a more targeted nutritional approach can be achieved.

\section{Acknowledgements}

None.

(D) Brooke Humphrey 0000-0003-1132-8901

\section{Declaration of interest}

None.

\section{Ethics statement}

Not applicable.

\section{Software and data repository resources}

None of the data were deposited in an official repository.

\section{References}

Anastasilakis CD, loannidis 0, Gkiomisi Al and Botsios D 2013. Artificial nutrition and intestinal mucosal barrier functionality. Digestion 88, 193-208.

Bach Knudsen KE, Hedemann MS and Lærke HN 2012. The role of carbohydrates in intestinal health of pigs. Animal Feed Science and Technology 173, 41-53.

Bailey M, Haverson K, Inman C, Harris C, Jones P, Corfield G, Miller B and Stokes C 2005. The influence of environment on development of the mucosal immune system. Veterinary Immunology and Immunopathology 108, 189-198.

Bernabucci U, Ronchi B, Lacetera N and Nardone A 2005. Influence of body condition score on relationships between metabolic status and oxidative stress in periparturient dairy cows. Journal of Dairy Science 88, 2017-2026.

Bian G, Ma S, Zhu Z, Su Y, Zoetendal EG, Mackie R, Liu J, Mu C, Huang R, Smidt H and Zhu W 2016. Age, introduction of solid feed and weaning are more important determinants of gut bacterial succession in piglets than breed and nursing mother as revealed by a reciprocal cross-fostering model. Environmental Microbiology 18, 1566-1577.

Bonaz BL and Bernstein CN 2013. Brain-gut interactions in inflammatory bowel disease. Gastroenterology 144, 36-49.

Brubaker PL and Anini Y 2003. Direct and indirect mechanisms regulating secretion of glucagon-like peptide-1 and glucagon-like peptide-2. Canadian Journal of Physiology Pharmacology 81, 1005-1012.

Buchet A, Belloc C, Leblanc-Maridor M and Merlot E 2017. Effects of age and weaning conditions on blood indicators of oxidative status in pigs. PLoS One 12, e0178487.

Burrin DG and Stoll B 2009. Metabolic fate and function of dietary glutamate in the gut. American Journal of Clinical Nutrition 90, 850S-856S.

Burrin DG, Stoll B and Guan X 2003. Glucagon-like peptide 2 function in domestic animals. Domestic Animal Endocrinology 24, 103-122.

Burrin DG, Stoll B, Jiang R, Chang X, Hartmann B, Holst JJ, Greeley GH Jr and Reeds PJ 2000. Minimal enteral nutrient requirements for intestinal growth in neonatal piglets: how much is enough? American Journal of Clinical Nutrition 71, 1603-1610.

Capozzalo MM, Kim JC, Htoo JK, de Lange CF, Mullan BP, Hansen CF, Resink JW, Stumbles PA, Hampson DJ and Pluske JR 2012. An increased ratio of dietary tryptophan to lysine improves feed efficiency and elevates plasma tryptophan and kynurenine in the absence of antimicrobials and regardless of infection with enterotoxigenic Escherichia coli in weaned pigs. Journal of Animal Science 90 (suppl. 4), 191-193.

Carroll JA, Gaines AM, Spencer JD, Allee GL, Kattesh HG, Roberts MP and Zannelli ME 2003. Effect of menhaden fish oil supplementation and lipopolysaccharide exposure on nursery pigs. I. Effects on the immune axis when fed diets containing spray-dried plasma. Domestic Animal Endocrinology 24, 341-351.

Castillo C, Hernandez J, Bravo A, Lopez-Alonso M, Pereira V and Benedito JL 2005. Oxidative status during late pregnancy and early lactation in dairy cows. Veterinary Journal 169, 286-292.

Catoni C, Peters A and Schaefer HM 2008. Life history trade-offs are influenced by the diversity, availability and interactions of dietary antioxidants. Animal Behaviour 76, 1107-1119.

Cera KR, Mahan DC and Reinhart GA 1988. Weekly digestibilities of diets supplemented with corn oil, lard or tallow by weanling swine. Journal of Animal Science 66, 1430-1437.

Demehri FR, Barrett M, Ralls MW, Miyasaka EA, Feng Y and Teitelbaum DH 2013. Intestinal epithelial cell apoptosis and loss of barrier function in the setting of altered microbiota with enteral nutrient deprivation. Front Cell Infection Microbiology 3, 105.

Everaert N, Van Cruchten S, Weström B, Bailey M, Van Ginneken C, Thymann T and Pieper R 2017. A review on early gut maturation and colonization in pigs, 
including biological and dietary factors affecting gut homeostasis. Animal Feed Science and Technology 233, 89-103.

Foltz M, Maljaars J, Schuring EA, van der Wal RJ, Boer T, Duchateau GS, Peters HP, Stellaard F and Masclee AA 2009. Intragastric layering of lipids delays lipid absorption and increases plasma CCK but has minor effects on gastric emptying and appetite. American Journal Physiology Gastrointestine Liver Physiology 296, G982-G991.

Goodband B, Tokach M, Dritz S, Derouchey J and Woodworth J 2014. Practical starter pig amino acid requirements in relation to immunity, gut health and growth performance. Journal Animal Science Biotechnology 5, 12.

Gregory PC, McFadyen M and Rayner DV 1989a. Control of gastric emptying in the pig: influence of duodenal infusions of glucose and emulsified fat. Quarterly Journal of Experimental Physiology 74, 109-119.

Gregory PC, McFadyen M and Rayner DV 1989b. Duodenal infusion of fat, cholecystokinin secretion and satiety in the pig. Physiology \& Behavior 45, 1021-1024.

Gregory PC and Rayner DV 1987. The influence of gastrointestinal infusion of fats on regulation of food intake in pigs. The Journal of Physiology 385, 471-481.

Gresse R, Chaucheyras-Durand F, Fleury MA, Van de Wiele T, Forano E and Blanquet-Diot S 2017. Gut microbiota dysbiosis in postweaning piglets: understanding the keys to health. Trends Microbiology 25, 851-873.

Hanczakowska E 2017. The use of medium-chain fatty acids in piglet feeding - a review. Annals Animal Science 17, 967-977.

Hansen KB, Rosenkilde MM, Knop FK, Wellner N, Diep TA, Rehfeld JF, Andersen UB, Holst JJ and Hansen HS 2011. 2-Oleoyl glycerol is a GPR119 agonist and signals GLP-1 release in humans. The Journal of Clinical Endocrinology and Metabolism 96, E1409-E1417.

Heo JM, Kim JC, Hansen CF, Mullan BP, Hampson DJ, Maribo H, Kjeldsen N and Pluske JR 2010a. Effects of dietary protein level and zinc oxide supplementation on the incidence of post-weaning diarrhoea in weaner pigs challenged with an enterotoxigenic strain of Escherichia coli. Livestock Science 133, 210-213.

Heo JM, Kim JC, Hansen CF, Mullan BP, Hampson DJ and Pluske JR 2009. Feeding a diet with decreased protein content reduces indices of protein fermentation and the incidence of postweaning diarrhea in weaned pigs challenged with an enterotoxigenic strain of Escherichia coli. Journal of Animal Science 87, 2833-2843.

Heo JM, Kim JC, Hansen CF, Mullan BP, Hampson DJ and Pluske JR 2010b. Feeding a diet with a decreased protein content reduces both nitrogen content in the gastrointestinal tract and post-weaning diarrhoea, but does not affect apparent nitrogen digestibility in weaner pigs challenged with an enterotoxigenic strain of Escherichia coli. Animal Feed Science and Technology 160, 148-159.

Htoo JK, Araiza BA, Sauer WC, Rademacher M, Zhang Y, Cervantes M and Zijlstra RT 2007. Effect of dietary protein content on ileal amino acid digestibility, growth performance, and formation of microbial metabolites in ileal and cecal digesta of early-weaned pigs. Journal of Animal Science 85, 3303-3312.

Hussein MO, Hoad CL, Wright J, Singh G, Stephenson MC, Cox EF, Placidi E, Pritchard SE, Costigan C, Ribeiro H, Ciampi E, Nandi A, Hedges N, Sanderson P, Peters HP, Rayment P, Spiller RC, Gowland PA and Marciani L (2015). Fat emulsion intragastric stability and droplet size modulate gastrointestinal responses and subsequent food intake in young adults. Journal of Nutrition $145,1170-1177$.

Jacobi SK and Odle J 2012. Nutritional factors influencing intestinal health of the neonate. Advances in Nutrition 3, 687-696.

Kim BG, Kil DY and Stein HH 2013. In growing pigs, the true ileal and total tract digestibility of acid hydrolyzed ether extract in extracted corn oil is greater than in intact sources of corn oil or soybean oil. Journal of Animal Science 91, 755-763.

Kim JC, Hansen CF, Mullan BP and Pluske JR 2012. Nutrition and pathology of weaner pigs: nutritional strategies to support barrier function in the gastrointestinal tract. Animal Feed Science and Technology 173, 3-16.

Kim JC, Mullan BP, Black JL, Hewitt RJ, van Barneveld RJ and Pluske JR 2016. Acetylsalicylic acid supplementation improves protein utilization efficiency while vitamin E supplementation reduces markers of the inflammatory response in weaned pigs challenged with enterotoxigenic $E$. coli. Journal Animal Science Biotechnology 7, 58.

Klasing KC and Johnstone BJ 1991. Monokines in growth and development. Poultry Science 70, 1781-1789.
Knapper JM, Heath A, Fletcher JM, Morgan LM and Marks V 1995. GIP and GLP-1(7-36)amide secretion in response to intraduodenal infusions of nutrients in pigs. Comparative Biochemistry Physiology C Pharmacology Toxicology Endocrinology 111, 445-450.

Kvidera SK, Horst EA, Sanz Fernandez MV, Abuajamieh M, Ganesan S, Gorden PJ, Green HB, Schoenberg KM, Trout WE, Keating AF and Baumgard LH 2017. Characterizing effects of feed restriction and glucagon-like peptide 2 administration on biomarkers of inflammation and intestinal morphology. Journal of Dairy Science 100, 9402-9417.

Lalles JP, Bosi P, Smidt H and Stokes CR 2007. Nutritional management of gut health in pigs around weaning. Proceeding of Nutrition Society 66, 260-268. Le Dividich J and Seve B 2000. Effects of underfeeding during the weaning period on growth, metabolism, and hormonal adjustments in the piglet. Domestic Animal Endocrinology 19, 63-74.

Le Floc'h N, Lebellego L, Matte JJ, Melchior D and Seve B 2009. The effect of sanitary status degradation and dietary tryptophan content on growth rate and tryptophan metabolism in weaning pigs. Journal of Animal Science 87, 1686-1694.

Lee YS and Jun HS 2016. Anti-inflammatory effects of GLP-1-based therapies beyond glucose control. Mediators Inflammation 2016, 3094642.

Lewis MC, Inman CF, Patel D, Schmidt B, Mulder I, Miller B, Gill BP, Pluske J, Kelly D, Stokes CR and Bailey M 2012. Direct experimental evidence that early-life farm environment influences regulation of immune responses. Pediatric Allergy Immunology 23, 265-269.

Li D, Xi P, Wang J, Wang J, Ren J, Kang Y and Thacker PA 2001. The effect of lysine to protein ratio on growth performance and efficiency of nitrogen utilization in pigs. Asian-Australian Journal of Animal Science 14, 1282-1289.

Li S, Sauer WC and Fan MZ 1993. The effect of dietary crude protein level on amino acid digestibility in early-weaned piglets. Journal of Physiology Animal Nutrition 70, 26-37.

Main RG, Dritz SS, Tokach MD, Goodband RD and Nelssen JL 2004. Increasing weaning age improves pig performance in a multisite production system. Journal of Animal Science 82, 1499-1507.

Marciani L, Faulks R, Wickham MS, Bush D, Pick B, Wright J, Cox EF, Fillery-Travis A, Gowland PA and Spiller RC 2009. Effect of intragastric acid stability of fat emulsions on gastric emptying, plasma lipid profile and postprandial satiety. British Journal of Nutrition 101, 919-928.

Marciani L, Wickham M, Singh G, Bush D, Pick B, Cox E, Fillery-Travis A, Faulks R, Marsden C, Gowland PA and Spiller RC 2007. Enhancement of intragastric acid stability of a fat emulsion meal delays gastric emptying and increases cholecystokinin release and gallbladder contraction. American Journal Physiology Gastrointestine Liver Physiology 292, G1607-G1613.

McCracken BA, Spurlock ME, Roos MA, Zuckermann FA and Gaskins HR 1999. Weaning anorexia may contribute to local inflammation in the piglet small intestine. Journal of Nutrition 129, 613-619.

Mereu A, Tedo G, Moeser AJ, Rimbach G and Ipharraguerre IR 2015. Cromolynmediated improvement of intestinal barrier function is associated with enhanced piglet performance after weaning. BMC Veterinary Research 11, 274.

Merrifield CA, Lewis MC, Berger B, Cloarec O, Heinzmann SS, Charton F, Krause L, Levin NS, Duncker S, Mercenier A, Holmes E, Bailey M and Nicholson JK 2016. Neonatal environment exerts a sustained influence on the development of the intestinal microbiota and metabolic phenotype. ISME Journal 10, 145-157.

Moeser AJ, Klok CV, Ryan KA, Wooten JG, Little D, Cook VL and Blikslager AT 2007. Stress signaling pathways activated by weaning mediate intestinal dysfunction in the pig. American Journal Physiology Gastrointestine Liver Physiology 292, G173-G181.

Moeser AJ, Pohl CS and Rajput M 2017. Weaning stress and gastrointestinal barrier development: implications for lifelong gut health in pigs. Animal Nutrition 3, 313-321.

Nemechek JE, Tokach M, Britz SS, Goodband B, DeRouchey J, Nelssen JL and Ursy $J$ 2011. Effect of total lysine:crude protein ratio on growth performance of nursery pigs from 15 to $25 \mathrm{lb}$. Proceedings of the Swine Industry Day, Kansas State University 1056, 70-78.

Nyachoti CM, Omogbenigun FO, Rademacher M and Blank G 2006. Performance responses and indicators of gastrointestinal health in early-weaned pigs fed low-protein amino acid-supplemented diets. Journal of Animal Science 84 125-134.

Orzechowski A, Ostaszewski P, Wilczak J, Jank M, Balasinska B, Wareski P and Fuller Jr 2002. Rats with a glucocorticoid-induced catabolic state show 


\section{Humphrey, Zhao and Faris}

symptoms of oxidative stress and spleen atrophy: the effects of age and recovery. Journal of Veterinary Medicine. A, Physiology, Pathology, Clinical Medicine 49, 256-263.

Opapeju FO, Krause DO, Payne RL, Rademacher M and Nyachoti CM 2009. Effect of dietary protein level on growth performance, indicators of enteric health, and gastrointestinal microbial ecology of weaned pigs induced with postweaning colibacillosis. Journal of Animal Science 87, 2635-2643.

Pastorelli H, van Milgen J, Lovatto P and Montagne L 2012. Meta-analysis of feed intake and growth responses of growing pigs after a sanitary challenge. Animal 6, 952-961.

Pieper R, Kroger S, Richter JF, Wang J, Martin L, Bindelle J, Htoo JK von Smolinski D, Vahjen W, Zentek J and Van Kessel AG 2012. Fermentable fiber ameliorates fermentable protein-induced changes in microbial ecology, but not the mucosal response, in the colon of piglets. Journal of Nutrition 142, 661-667.

Pluske J, Hampson DJ and Williams IH 1997. Factors influencing the structure and function of the small intestine in the weaned pig: a review. Livestock Production Science 51, 215-236.

Pluske JR 1993. Psychological and nutritional stress in pigs at weaning: production parameters, the stress response, and histology and biochemistry of the small intestine. The University of Western Australia, Perth, WA, Australia.

Ramayo-Caldas Y, Mach N, Lepage $P$, Levenez F, Denis C, Lemonnier G, Leplat JJ, Billon Y, Berri M, Dore J, Rogel-Gaillard C and Estelle J 2016. Phylogenetic network analysis applied to pig gut microbiota identifies an ecosystem structure linked with growth traits. ISME Journal 10, 2973-2977.

Shi J, Yu J, Pohorly JE and Kakuda Y 2003. Polyphenolics in grape seeds biochemistry and functionality. Journal of Medicinal Food 6, 291-299.

Steingoetter A, Buetikofer S, Curcic J, Menne D, Rehfeld JF, Fried M Schwizer W and Wooster TJ 2017. The dynamics of gastric emptying and self-reported feelings of satiation are better predictors than gastrointestinal hormones of the effects of lipid emulsion structure on fat digestion in healthy adults-a Bayesian inference approach. Journal of Nutrition 147, 706-714.

Stoll B, Henry J, Reeds PJ, Yu H, Jahoor F and Burrin DG 1998. Catabolism dominates the first-pass intestinal metabolism of dietary essential amino acids in milk protein-fed piglets. Journal of Nutrition 128, 606-614.
Sun Y and Kim SW 2017. Intestinal challenge with enterotoxigenic Escherichia coli in pigs, and nutritional intervention to prevent postweaning diarrhea. Animal Nutrition 3, 322-330.

Tokach MD, Goodband RD, Nelssen JL and Kats LJ 1992. Influence of weaning weight and growth during the first week postweaning on subsequent pig performance. Swine Day 14-17.

Urbaityte R, Mosenthin R, Eklund M, Piepho HP and Rademacher M 2009 Determination of standardized ileal crude protein and amino acid digestibilities in protein supplements for piglets. Animal 3, 1696-1705.

Voortman T, Hendriks HF, Witkamp RF and Wortelboer HM 2012. Effects of longand short-chain fatty acids on the release of gastrointestinal hormones using an ex vivo porcine intestinal tissue model. Journal Agriculture Food Chemistry 60 9035-9042.

Wang W, Zeng X, Mao X, Wu G and Qiao S 2010. Optimal dietary true ileal digestible threonine for supporting the mucosal barrier in small intestine of weanling pigs. Journal of Nutrition 140, 981-986.

Wei HK, Xue HX, Zhou ZX and Peng J 2017. A carvacrol-thymol blend decreased intestinal oxidative stress and influenced selected microbes without changing the messenger RNA levels of tight junction proteins in jejunal mucosa of weaning piglets. Animal 11, 193-201.

Wijtten PJ, van der Meulen J and Verstegen MW 2011. Intestinal barrier function and absorption in pigs after weaning: a review. The British Journal of Nutrition 105, 967-981.

Williams BA, Verstegen MW and Tamminga S 2001. Fermentation in the large intestine of single-stomached animals and its relationship to animal health. Nutrition Research Reviews 14, 207-228.

Wu X, Chen D, Yu B, Luo Y, Zheng P, Mao X, Yu J and He J 2018. Effect of different dietary non-starch fiber fractions on growth performance, nutrient digestibility, and intestinal development in weaned pigs. Nutrition 51-52, 20-28.

Xu J, Xu C, Chen X, Cai X, Yang S, Sheng Y and Wang T 2014. Regulation of an antioxidant blend on intestinal redox status and major microbiota in early weaned piglets. Nutrition 30, 584-589.

Zeng MY, Onohara N and Nunez G 2017. Mechanisms of inflammation-driven bacterial dysbiosis in the gut. Mucosal Immunology 10, 18-26.

Zhu LH, Zhao KL, Chen XL and Xu JX 2012. Impact of weaning and an antioxidant blend on intestinal barrier function and antioxidant status in pigs. Journal of Animal Science 90, 2581-2589. 